УДК 537.874 .4

\title{
Mathematical Models of the Motion of Submersible Apparat with Electromagnetic Expiation and Reception near the Sea Bottom
}

\author{
Georgy Ya. Shaidurov \\ Galina N. Romanova \\ Danil S. Kudinov* \\ Military Engineering Institute \\ Siberian Federal University \\ Akademgorodok, 13A/8, Krasnoyarsk, 660036
}

Russia

Received 21.01.2017, received in revised form 22.03.2017, accepted 20.05.2017

\begin{abstract}
We give aqualitative assessment of the dispersion of the received electromagnetic interference due to variations in the electrical conductivity of the medium and the distance to the soil when search vehicle driving near these a water-soil boundary, we determine the best modes of motion and parameters of the probing signal for different transmitter-receiver combinations.
\end{abstract}

Keywords: submarine, sea water, reflected wave, electromagnetic, antenna, interference, boundary. DOI: 10.17516/1997-1397-2017-10-4-522-530.

\section{Introduction}

In applied problems for electromagnetic sensing of marine soil, to create mobile machines and robots to search for various natural and artificial objects in sea water, it is of interest to estimate sea soil reaction and the environment, as well as the random movement factors to the observed response signals during pulse or harmonic form of electrical excitation, or magnetic dipoles.

In [1-4] discusses the various theoretical aspects of harmonic and impulse waves in a conductive medium, including the two-layer model [3], however, for use in the calculation of limit search characteristics determined by the general movement interference mathematical relationships don't give much. This article discusses the problem of search of small-size conductive bodies embedded in the bottom ground, using a deep-sea vehicle with a small carrier base where the transmitter and receiver adjacent to each other on the same carrier when a powerful primary field emitter on the $80-100 \mathrm{~dB}$ exceeds received signal.

Under these conditions, the main limitation on the resolution of the device on the allocation of weak anomalies play a so-called synchronous noise associated with the fluctuations of the transmitter and receiver locations geometry, variations in the height of the device movement over the water-soil boundary and changes in the electrical conductivity of the two medium.

An important role plays the usage of type of electromagnetic transmitter and receiver: electric, magnetic, or a mixed type, as well as the shape of the probe signal - a harmonic or pulse with appropriate algorithms release their information options.

Statement of the problem reduces to evaluating the dispersion synchronous interference (SI) occurring at the search vehicle electromagnetic receiver input when carrier moves the near the seabed by apparatuses movement height variations above the ground, relative conductivities

*kudinovdanil@yandex.ru

(c) Siberian Federal University. All rights reserved 
of the environment for different combinations of transmitting and receiving electromagnetic or magnetic fields, radiated signals parameters determination, travel heights, and the optimal combination of transmitting and receiving antennas minimized SI.

The [5-7] describes the features of the theory and application of electromagnetic search systems in sea water with the harmonic probe signal, and $[3,8]$ with the pulse signal. However, the influence of movement disturbing factors is not determined as well as the features of the pulse variant environment sensing.

This article provides estimates of the expected signal and synchronous interference of search vehicle for both type of the probing signal.

\section{Calculation data}

We take the boundary between the medium as flat infinite along strike, medium parameters respectively, $\sigma_{1}, \mu_{1}, \epsilon_{1}$, and $\sigma_{2}, \mu_{2}, \epsilon_{2}$. We neglect the displacement currents. A dipole source is located in the water. Further in the first medium at 0 point at a height $\mathrm{H}$ from the border, the receiver at $O_{1}$ point at height $z$. The receiver coordinates in cylindrical system $(z, \rho, \phi)$, Fig. 1 .

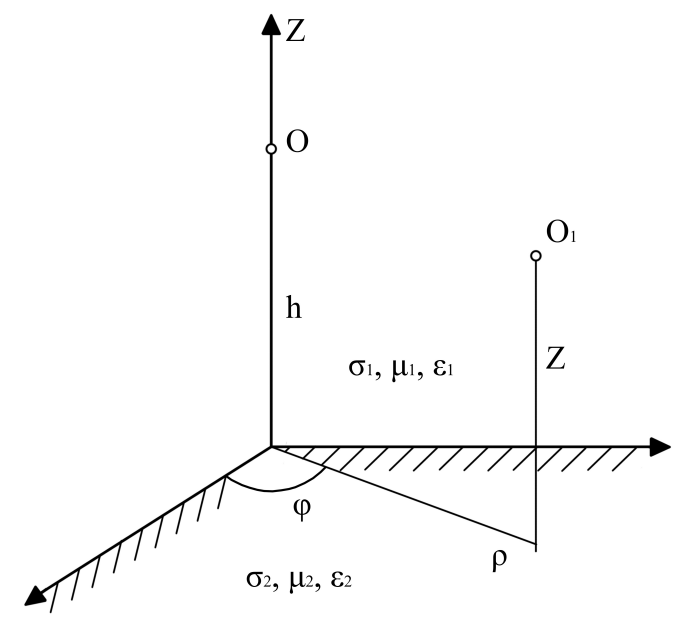

Fig. 1. Receiver in cylindrical coordinates system

Consider the observed interference signals for different excitation systems and receiving except the top of the marine environment boundaries, i.e. consider that search vehicle moves near the ground at great depths of $100 \mathrm{~m}$. and deeper, so that the electron-magnetic waves are completely attenuated before surface.

We find electric and magnetic fields at the receiving point through electric potentials [9] using the equations:

$$
\begin{gathered}
h E=i \omega \mu_{1} \vec{A}^{E}+\frac{1}{\sigma} \cdot \operatorname{grad} \operatorname{div} \vec{A}^{E}, \\
E=\omega \vec{A}^{H}+\frac{1}{i \omega \mu_{1}} \cdot \operatorname{grad} \operatorname{div} \vec{A}^{H} .
\end{gathered}
$$

The components of the observed electrical and magnetic fields could be written in terms of the electric and magnetic numbers:

$$
\begin{gathered}
E_{x, y, z}^{E, H}=\Gamma\left(e_{x, y, z}^{E, H}\right) \\
H_{x, y, z}^{E, H}=\Gamma\left(h_{x, y, z}^{E, H}\right),
\end{gathered}
$$


where

$$
\begin{aligned}
& A_{x}^{E}=\frac{P_{x}}{4 \pi} \int_{0}^{\infty} \frac{\lambda}{m_{1}} I_{0}(\lambda \rho) \alpha^{H} e^{-m_{1}(z+h)} d \lambda, \\
& A_{z}^{E}=\frac{P_{x}}{4 \pi} \int_{0}^{\infty} \frac{1}{\lambda} I_{0}(\lambda \rho)\left[\alpha^{E}+\alpha^{H}\right] e^{-m_{1}(z+h)} d \lambda,
\end{aligned}
$$

magnetic dipoles with moments $M_{x}$ and $M_{y}$ :

$$
\begin{aligned}
& A_{x}^{H}=\frac{i \omega \mu_{1} M_{x}}{4 \pi} \int_{0}^{\infty} \frac{\lambda^{2}}{m_{1}} I_{1}(\lambda \rho) \alpha^{H} \sin \phi e^{-m_{1}(z+h)} d \lambda, \\
& A_{y}^{H}=\frac{i \omega \mu_{1} M_{y}}{4 \pi} \int_{0}^{\infty} \frac{\lambda^{2}}{m_{1}} I_{1}(\lambda \rho) \alpha^{H} \cos \phi e^{-m_{1}(z+h)} d \lambda,
\end{aligned}
$$

where $\alpha^{H}$ and $\alpha^{E}$ is the reflection coefficients for magnetic and electric field type; $P_{x}=I \cdot L$ and $M=I \cdot S_{\Gamma}$ is the electric and magnetic dipole moment.

$$
\alpha^{H}=\frac{m_{1}-m_{2}}{m_{1}+m_{2}}, \quad \alpha^{E}=\frac{m_{1} \sigma_{2}-m_{2} \sigma_{1}}{m_{1} \sigma_{2}+m_{2} \sigma_{1}}, \quad m_{1}=\sqrt{\lambda^{2}-K_{1}^{2}}, \quad m_{2}=\sqrt{\lambda^{2}-K_{2}^{2}},
$$

where $K_{1}^{2}=1 \omega \mu_{1} \sigma_{1}$ and $K_{2}^{2}=1 \omega \mu_{2} \sigma_{2}$ are wave number of media; $I_{0}(\alpha \rho)$ and $I_{1}(\alpha \rho)$ are Bessel functions; $\quad \rho=\sqrt{x^{2}+y^{2}}$ is the projection of the distance between the transmitter and the receiver on the plane $X Y$.

$$
\begin{aligned}
& \Gamma_{E}=\frac{1}{4 \pi} \int_{0}^{\infty} e_{x, y, z}^{E, H} \exp \left[-m_{1}(z+h)\right] d x, \\
& \Gamma_{H}=\frac{1}{4 \pi} \int_{0}^{\infty} h_{x, y, z}^{E, H} \exp \left[-m_{1}(z+h)\right] d x,
\end{aligned}
$$

where $\Gamma$ is electric or magnetic type operator.

In particular,for the $E E_{X} / M R_{Y}$ set, i.e. electric excitation and magnetic reception:

$$
h_{x, y}^{E}=-P\left[\lambda I_{0}(\lambda \rho) \alpha^{H}+\lambda\left[\frac{I_{1}(\lambda \rho)}{\lambda \rho}\left(\cos ^{2} \phi-\sin ^{2} \phi\right)-I_{0}(\lambda \rho) \cos ^{2} \phi\right]\left(\alpha^{H}+\alpha^{E}\right)\right] .
$$

for the $E E_{X} / M R_{Z}$ set

$$
h_{x, z}^{E}=P \frac{\lambda^{2}}{m_{1}} I_{1}(\lambda \rho) \alpha^{H} \sin \phi
$$

for the $M E_{X} / M R_{X}$ set (magnetic excitation/magnetic reception)

$$
h_{x x}^{H}=M\left[\frac{K_{1} \lambda}{m_{1}} I_{0}(\alpha \rho) \alpha^{E}+\left[\frac{I_{1}(\alpha \rho)}{\alpha \rho}\left(\cos ^{2} \phi-\sin ^{2} \phi\right)-I_{0}(\lambda \rho) \cos ^{2} \phi\right]\left(\frac{K_{1}^{2}}{m_{1}} \alpha^{E}-m_{1} \alpha^{H}\right)\right] .
$$

The component fields of the $P_{y}$ electric dipole can be determined from the equation for $P_{x}$, by reversing the $x$ and $y$ locations and $\sin \phi$ and $\cos \phi$ locations. Similarly, we obtain formulas for the $H_{y}$ from $H_{z}$. Time functions are found by applying the equation $(7,8,9)$, inverse discrete Fourier transform for the particular shape of the probing signal(PS).

Fig. 2 shows major types of temporal characteristics of the two-layer medium reaction on an electrical stimulation or a magnetic dipole with a current in the form of a sequence of alternating half-sine pulse with a complex spectral density:

$$
I(i, \omega)=2 I_{m} \frac{\frac{\pi}{\tau_{u}}}{\left(\frac{\pi}{\tau_{u}}\right)^{2}-\left(\frac{2 \pi n}{T}\right)^{2}}\left(1+e^{-i \frac{2 \pi n}{T} \tau_{u}}\right),
$$


where $\tau_{u}$ is impulse duration, $T$ is recurrence interval, $n=1,3,5 \ldots$ The first medium is sea water, the second is ground.

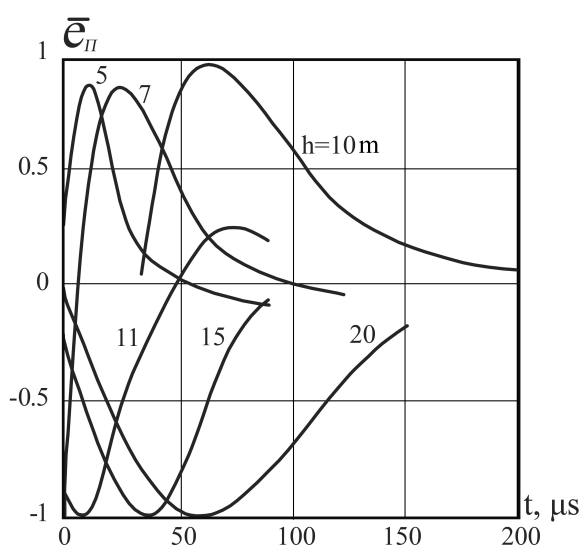

Fig. 2. Time-response characteristic of the two-layer medium reaction for the $E E_{X} / M R_{Y}$ system $\sigma_{1}=1 \mathrm{~S} / \mathrm{m}, \sigma_{2}=0.1 \mathrm{~S} / \mathrm{m}, \tau_{u}=50 \mu \mathrm{s}, t=100 \mu \mathrm{s}$

\section{The simulation results}

Calculations were carried out numerically for individual moments of excitation and reception $I_{m} L S_{R}=1, I_{m} S_{\Gamma} S_{R}=1$. The normalization of the timing determined by the maximum value for each height $h$. At a constant conductivity of the medium time signal delay depends only on the height of the set position on the ground Fig. 2, i. e, it is determined by the group delay time of the signal at the $\mathrm{t}$ emitter-ground-receiver site. For a fixed point of reference $t=100 \mu \mathrm{s}$ dependence on the parameter $h$ has some extremes Fig. 3. This fact gives hope to receive additional control factor to reduce the influence of variations in ground elevation. The dependence of the signal amplitude on the electrical conductivity of the soil Fig. 4 has a monotonous character. For small ratio $\sigma_{2} / \sigma_{1}$ amplitude increases faster and in $\sigma_{2} / \sigma_{1}>0.7$ curve of this relationship comes to an asymptote, which corresponds to the set work in a homogeneous medium.

On the assumption of smallness relative increments of variable parameters of the $\Delta h / h \ll 1$, $\sigma_{2} / \sigma_{1}$ interface, the RMS value of the amplitude of the synchronous interference will be found from the equations:

$$
\tilde{e}_{h}=\sqrt{D_{h}}=\left(G_{h} \sigma_{h}^{2}\right)^{1 / 2}, \quad \tilde{e}_{\sigma_{2}}=\sqrt{D_{\sigma_{2}}}=\left(G_{\sigma} \sigma_{\sigma_{2}}^{2}\right)^{1 / 2},
$$

where $D_{h}$ and $D_{\sigma 2}$ is the variance (power) of the synchronous interference on the parameters $h$ and $\sigma ; \sigma_{h}^{2}$ and $\sigma_{\sigma_{2}}^{2}$ is the dispersion terrain height and electrical conductivity;

$G_{h}=\frac{1}{\Delta h} \int_{e}^{h+\Delta h}\left(\frac{\partial e_{\Pi}}{\partial_{h}}\right)^{2} d h$ is average power gradient of the synchronous interference for the increment of height $h ; G_{\sigma_{2}}=\left(\frac{\partial e_{\Pi}}{\partial \sigma_{2}}\right)^{2}$ is the increment $\sigma_{2}$.

Temporary reaction function of the interface and the encompass medium:

$$
e_{\Pi}(t)=\Phi^{-1}\left[-i \omega \mu_{1} \Gamma\left(h_{x, y, z}^{E, H}\right)\right]
$$




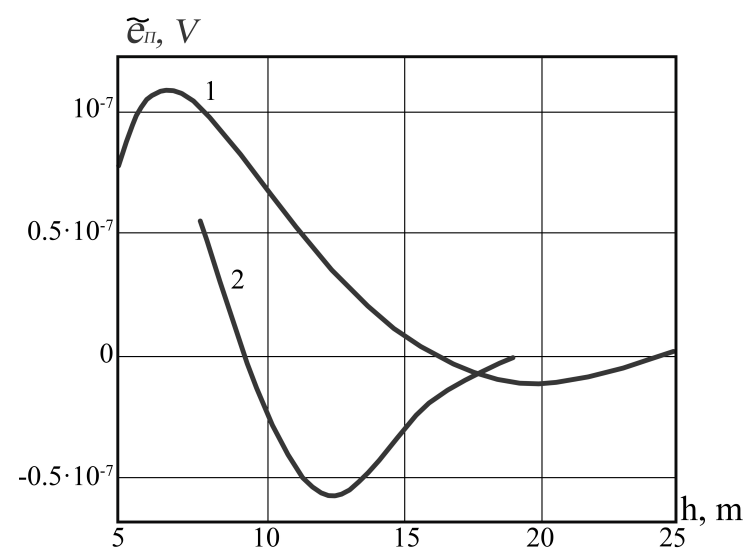

Fig. 3. Function of SI $\left(\tilde{e}_{\Pi}\right)$ vs the distance from the soil $h$. Here 1 is the $E E_{X} / M R_{Y}$ system, 2 is the $M E_{X} / M R_{Y}$ system, $\sigma_{1}=1 S / m, \sigma_{2}=0.1 S / m, \tau_{u}=50 \mu s, t=100 \mu s$

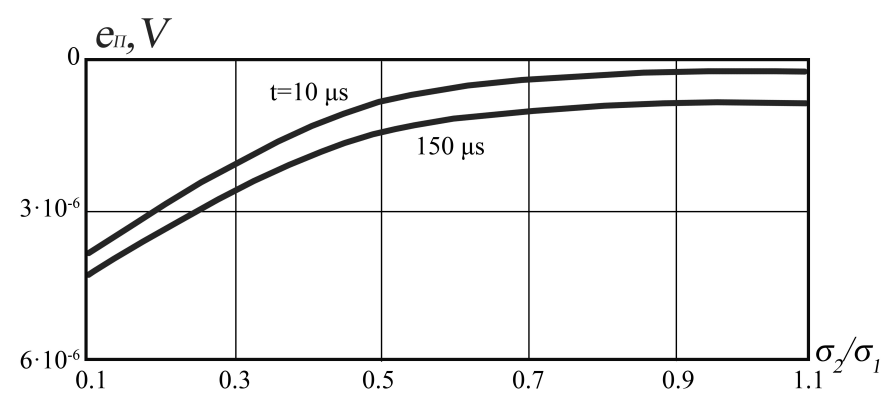

Fig. 4. Functional relation of signal amplitude vs electrical conductivity of the soil. Where $h=10 \mathrm{~m}, \sigma_{1}=1 \mathrm{~S} / \mathrm{m}, \tau_{u}=50 \mu \mathrm{s}$

where $\Phi^{1}$ is inverse Fourier transform operator.

The instantaneous value of the gradient and height of soil conductivity for $E E_{X} / M R_{Y}$ set:

$$
\begin{aligned}
& \left(\frac{\partial e_{\Pi}(t)}{\partial h}\right)^{2}=\left\{\Phi^{-1} \frac{\partial}{\partial h}\left[-i \omega \mu_{1} I(i \omega) \Gamma\left(h_{x, y}^{E}\right)\right]\right\}^{2}, \\
& \left(\frac{\partial e_{\Pi}(t)}{\partial \sigma_{2}}\right)^{2}=\left\{\Phi^{-1} \frac{\partial}{\partial \sigma^{2}}\left[-i \omega \mu_{1} I(i \omega) \Gamma\left(h_{x, y}^{E}\right)\right]\right\}^{2} .
\end{aligned}
$$

The estimation of synchronous interference capacity of other set types is the same. Typical dependence $G_{h}$ ratio on the height for the synchronous interference of half-sine pulse with $\tau_{u}=$ $50 \mu s$ duration, single point $P_{x}=1$ and fixed delay time in synchronous interference amplitude is shown in Fig. 5. The Fig. 6 constructs such dependence of $G_{\sigma_{2}}$ coefficient with electrical conductivity of the soil at an average $\sigma_{2}=0.3 \mathrm{~S} / \mathrm{m}$.

The areas of these minima factors correspond to the optimal heights recommended for movement of the set. The final movement mode choice be based on similar relationships for the signal/noise ratio. Compared with the effect of $h$ changes the impact of changes of soil electrical conductivity on the impulse response, because of the shielding properties of the water, not so much Fig. 7. The tenfold changes of $\sigma_{2}$ parameters in range of $50 \ldots 200 \mu s$ response amplitude 


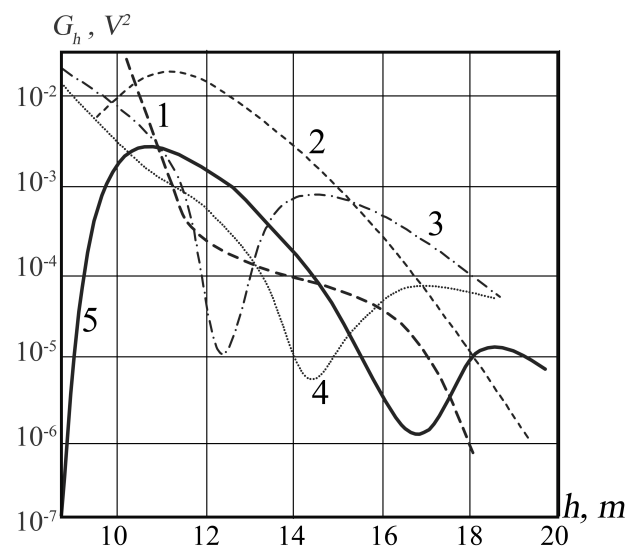

Fig. 5. Functional relation of the SI random component vs distance to the soil at the fixed time delay. Where 1 is $t=0 \mu \mathrm{s}, 2$ is $t=50 \mu \mathrm{s}, 3$ is $t=100 \mu \mathrm{s}, 4$ is $t=150 \mu \mathrm{s}, 5$ is $t=200 \mu s, \sigma_{1}=2 \mathrm{~S} / \mathrm{m}, \sigma_{2}=0.1 \mathrm{~S} / \mathrm{m}, \tau_{u}=50 \mu \mathrm{s}$

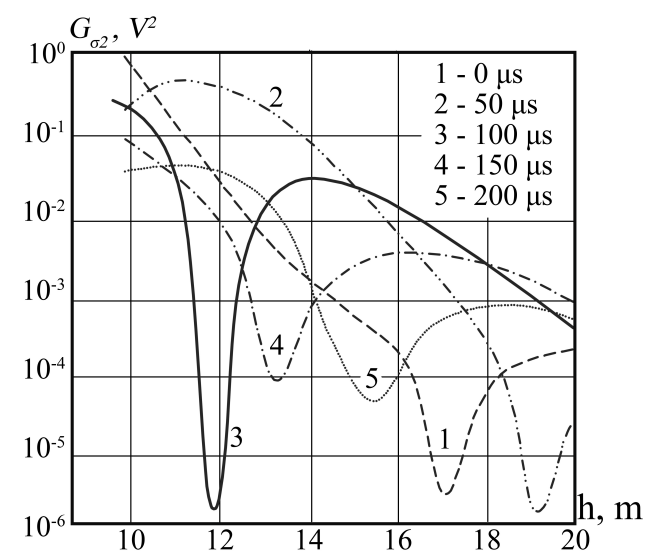

Fig. 6. Functional relation of the SI random component $G_{\sigma_{2}}$ vs distance to the soil at fixed time delay. Where $\sigma_{1}=2 \mathrm{~S} / \mathrm{m}, \sigma_{2}=0.3 \mathrm{~S} / \mathrm{m}, \tau_{u}=50 \mu \mathrm{s}$

changes by an average of $5 \%$. The maximum slope of the dependence $e_{\Pi}\left(\sigma_{2}\right)$ falls on soil and water high conductivity contrast plot $\sigma_{2} / \sigma_{1}>0.5$, but in the real such a sharp contrast is hardly possible due to upper soil layers saturation with water. It is interesting to compare these relationships with those in the case of amplitude change of $e_{\Pi}$ and phase change $\phi_{\Pi}$ synchronous interference harmonic form, Fig. 8 a, b. As in the case of pulsed excitation, there is an exponential dependence of EMF amplitude in the receiving antenna on distances to the ground and on its linear phase. The interface makes additional correction to the field in a uniform medium with $10 \%$, if $h=10 \mathrm{~m}$ and $0.2 \%$ for $h=20 \mathrm{~m}$. Increment of $h$ up to $1 \mathrm{~m}$ gives a phase shift of $16^{\circ}$ regardless of the type of excitation. The increment of the electrical conductivity of the soil $\sigma_{2}$ up to $1 / \mathrm{m}$ gives the phase change of 0.8 in e-mode and $3.3 \mathrm{in} \mathrm{m-mode.}$

RMS phase increment due to joint changes the parameters $h$ and $\sigma_{2}$ :

$$
\Delta \tilde{\phi}_{n}=\left\{\left[\frac{\partial}{\partial h} \operatorname{arctg}\left(\frac{I_{m} l_{x y}}{R e l_{x y}}\right) \Delta \tilde{h}\right]^{2}+\left[\frac{\partial}{\partial \sigma_{2}} \operatorname{arctg}\left(\frac{I_{m} l_{x y}}{R e l_{x y}} \Delta \tilde{\sigma}_{n}\right)\right]^{2}\right\}^{1 / 2},
$$




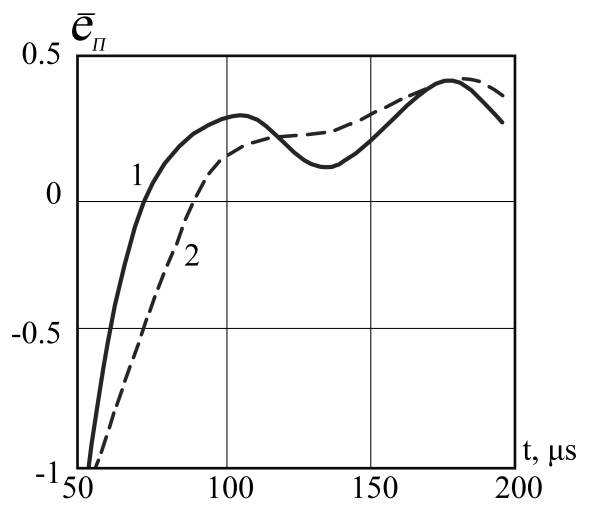

Fig. 7. Function of the impulse response vs ground electrical conductivity. Where $\sigma=2 S / m$, 2 is $\sigma_{2}=1.1 \mathrm{~S} / \mathrm{m}$
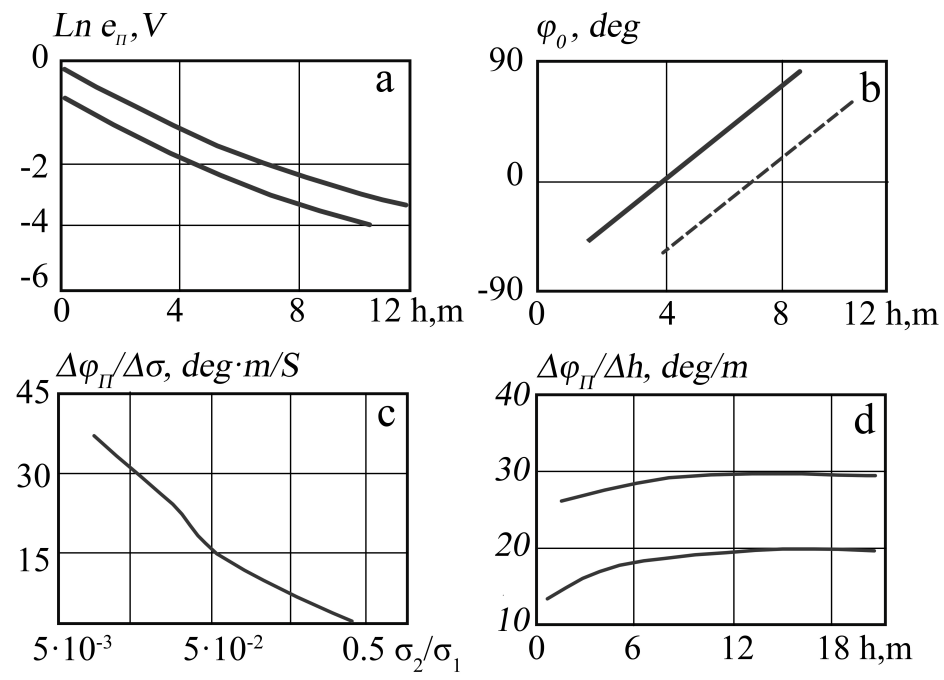

Fig. 8. a, b. Function of the amplitude and phase of the reflected signal vs source height at probing harmonic signal. Where 1 is the $M E_{X} / M R_{X}$ system, 2 is the $E E_{X} / M R_{Y}$ system. $\mathrm{c}, \mathrm{d}$. Function of phase gradient at synchronous interference vs the height and the ground conductivity. Where 1 is $f=10^{4} \mathrm{~Hz}, 2$ is $f=2.5 \cdot 10^{5} \mathrm{~Hz}$

where $I_{m} l_{x}, y, \operatorname{Re}_{x}, y$ is the reactive and active components of the interference $l_{x} y ; \Delta \tilde{h}$ is height standard deviation in the movement; $\Delta \tilde{\sigma}_{2}$ the mean square deviation of the ground-conductivity.

Numerical analysis of the equation (14) shows that in $E E_{X} / M R_{Y}$ system synchronous interference gradients phase and soil conductivity Fig. $8 \mathrm{c}$ are monotonic functions. The gradient $\Delta \phi / \Delta h$ is practically determined by the wave number of the upper half-space $\beta=R e^{\dot{K}_{1}}$.

Fig. $8 \mathrm{~d}$ shows that the $\Delta \phi_{\Pi} / \Delta h$ deviation from $2 \beta$ is $5-60^{\circ}$ at low altitudes $\left(h=1 \ldots 5_{m}\right)$ and tends to zero when $h>10 \mathrm{~m}$, i.e. $\Delta \phi_{\Pi} / \Delta h$ does not depend on the height average values. The gradient is defined by $\sigma_{2} / \sigma_{1}$ and if $\sigma_{2} / \sigma_{1} \rightarrow 1$ tends to zero. If we consider that the expected phase increment due to the object search is a value comparable to the relative level of the secondary field, i.e. about $10^{-6} \mathrm{rad}$, the increment of $\Delta \phi_{\Pi} / \Delta h$ and $\Delta \phi_{\Pi} / \Delta \sigma_{2}$ are extremely large, and the monotonic character of dependencies $\phi_{\Pi}(h)$ and $\phi_{\Pi}\left(\sigma_{2}\right)$ does not give hope for the 
choice of the optimal mode of movement, minimizing the geological interference, if the range of operating frequencies will not be expanded.

Among possible modifications of search systems exist sets are insensitive to a change in the coordinate parameters of synchronous interference in which any component of the secondary field is zero. Such systems will be called invariant (Tab. 1). Unfortunately, invariance is maintained only when moving in a very specific direction. For example, in $E E_{X} / M R_{Z}$ vertical movement of the carrier relative to the ground do not change the level of the synchronous interference, however, minor variations in the angular directions of the system led it into the category of noninvariant. However, according to the theory and the experimental setup invariant sets provide substantially smaller synchronous interference value.

Table 1. Combinations of radiation receiving-installations

\begin{tabular}{|c|c|c|}
\hline $\begin{array}{l}\text { Type of trans- } \\
\text { mission }\end{array}$ & Reflect field & $\begin{array}{l}\text { Invariant receiving } \\
\text { system detection }\end{array}$ \\
\hline 1 & 2 & 3 \\
\hline \multirow[t]{2}{*}{$E E_{x}$} & $H_{x x}=0 ; H_{x y}=0 ; H_{x z}=0 ; E_{x x} ; E_{x y} ; E_{x z}$ & $\begin{array}{l}M R_{z} ; \quad M R_{y} ; M R_{x} ; \\
\text { XOZ-plane }\end{array}$ \\
\hline & $H_{x x}=0 ; H_{x y} ; H_{x z} ; E_{x x} ; E_{x y}=0 ; E_{x z}=0$ & $\begin{array}{l}E R_{y} ; \quad E R_{z} ; M R_{x} \\
\text { YOZ-plane }\end{array}$ \\
\hline \multirow[t]{2}{*}{$M E_{z}$} & $H_{z x} ; H_{z y}=0 ; H_{z z} ; E_{z x} ; E_{z y}=0 ; E_{z z}=0$ & $\begin{array}{l}M R_{y} ; \quad E R_{z} ; E R_{x} ; \\
\text { XOZ-plane }\end{array}$ \\
\hline & $H_{z x}=0 ; H_{z y}=0 ; H_{z z} ; E_{z x} ; E_{z y}=0 ; E_{z z}=0$ & $\begin{array}{l}M R_{x} ; E R_{y} ; E R_{z} ; \\
\text { YOZ-plane }\end{array}$ \\
\hline \multirow[t]{2}{*}{$M E_{x}$} & $H_{x x}=0 ; H_{x y} ; H_{x z} ; E_{x x} ; E_{x y}=0 ; E_{x z}=0$ & $\begin{array}{l}M R_{y} ; E R_{x} ; E R_{y} ; \\
\text { XOZ-plane }\end{array}$ \\
\hline & $H_{x x} ; H_{x y}=0 ; H_{x z}=0 ; E_{x x}=0 ; E_{x y} ; E_{x z}$ & $\begin{array}{l}M R_{y} ; \quad M R_{z} ; E R_{x} ; \\
\text { YOZ-plane }\end{array}$ \\
\hline
\end{tabular}

\section{Conclusion}

We obtained essentially nonlinear dependence of the observed electromagnetic signals of search machines on the height position above the seabed and the relative conductivity of the host medium (water) and soil. This random signal component (synchronous interference) defined by variation of the height of the movement apparatus over the ground, depending on the duration of the probe pulse is minimized by determining the distance to the ground.

In the case of using the harmonic probe signal with a phase method of receiver processing dependence of synchronous interference (SI) on these factors is monotonic and the absolute level of SI dispersion significantly higher compared with the pulse sensing, indicating that the advantage of using the pulse-shaped radiation along with receiving signals in the pauses between the relatively strong primary field, which is not implemented in a harmonic signal sets.

The work was supported by RFBR 14-07-00187A grant "Research electromagnetic effects management of sea water parameters using ultrasound to improve radio communication equipment with underwater objects". 


\section{References}

[1] L.M.Brekhlovskikh, Reflection and Refraction of Spherical Waves, Uspehi Fiz. Nauk, 38(1949), no. 1, 1-42 (in Russian).

[2] E.Gerjuoy, Total reflection of waves from a point source, Communication on Pure and Applied Mathematics, 6(1953), no. 1, 73-91.

[3] A.T.Dehoop, Pulsed electromagnetic radiation from a line sources a two-media configuration, RadioNauka, 141979, no. 2, 253-268 (in Russian).

[4] L.M.Brekhovskikh, Waves in layered media, Moscow, Nauka, 1973 (in Russian).

[5] M.B.Shneerson, A.M.Lungin, Pulsed electromagnetic research system, KSTU, 1990 (in Russian).

[6] N.I.Kalashnikov, F.L.Dudkin, Y.B.Nikolaenko, Fundamentals of marine electrical exploration, Naukova Dumka, 1980 (in Russian).

[7] V.I.Gordienko, Marine geophysical exploration, Naukova Dumka, 1987 (in Russian).

[8] J.A.Burrell, L.Peters, Distribution of low-frequency video pulses in lossy medium, EPIEE, 67(1979), no. 7, 6-17 (in Russian).

[9] B.S.Svetov, Theory, methodology and interpretation of materials of a low-frequency induction survey, Nedra, 1973 (in Russian).

\section{Математические модели движения подводного аппарата с электромагнитным излучением и приемом над морским дном}

Георгий Я ІШайдуров

Галина Н. Романова

Данил С. Кудинов

Военно-инженерный институт Сибирский федеральный университет Академгородок, 13А, корпус 8, 660036

Россия

Дается качественная оченка дисперсии принимаемых электромагнитных помех за счет вариаиии электропроводности средь и расстояния до грунта при движении поискового аппарата вблизи границы раздела морская вода-грунт, определяются оптимальные режимы движения и параметры излучаемых зондирующих сигналов для различных комбинаций излучатель-приемник.

Ключевые слова: подводная лодка, морская вода, отраженная волна, электромагнитный, антенна, помеха, граница. 